\title{
mTOR-Inhibition
}

\section{Temsirolimus für das Mantelzell-Lymphom zugelassen}

\section{Mit dem mTOR-Inhibitor Temsirolimus wurde jetzt erstmals ein Wirkstoff speziell zur Behandlung des rezidivierten und/oder refraktären Mantelzell-Lymphoms zugelassen.}

Die Zulassung beruht auf den Ergebnissen einer Phase-III-Studie bei 162 Patienten mit fortgeschrittenem Mantelzell-Lymphom (MCL), die bereits zwei bis sieben Vortherapien erhalten und somit alle Therapiealternativen ausgeschöpft hatten, so Georg Heß, Mainz. Bei diesen Patienten führte Temsirolimus (Torisel $^{\circledR}$ ) nicht nur zu einer hoch signifikant höheren Ansprechrate (mit 22 vs. $2 \%$ ), auch das progressionsfreie Überleben (PFS) war hoch signifikant von 1,9 auf 4,8 Monate verlängert. Die PFS-Verlängerung war dabei unabhängig vom Alter, Karnofsky-Status, von der Zahl der Vortherapien und der jeweiligen MCL-Variante. Beim Gesamtüberleben war allerdings nur trendmäßig ein Vorteil in der Gruppe der Patienten, die mit
Temsirolimus behandelt worden waren, zu verzeichnen. Ihre Überlebenszeit betrug 12,8 vs. 9,7 Monate bei Patienten, die eine Chemotherapie aus vorgegebenen Monotherapien (Gemcitabin i.v. oder Fludarabin i.v.)

\section{Kurz notiert}

erhielten. Die Behandlung mit Temsirolimus wurde laut $\mathrm{He}$ gut toleriert, die auftretenden Nebenwirkungen waren gut beherrschbar.

\section{CV}

Quelle: Pressegespräch und Mittagssymposium am 24. Februar 2010 beim 29. Deutschen Krebskongress in Berlin; Veranstalter: Wyeth Pharma
Experten plädieren für bessere schmerztherapeutische Versorgung von Krebspatienten Im Zuge des zweiten bundesweiten Aktionstages Tumorschmerz in Limburg machte der DGSS (Arbeitskreis Tumorschmerz der Deutschen Gesellschaft zum Studium des Schmerzes e.V.) auf die mangelende schmerztherapeutische Versorgung von Krebspatienten aufmerksam. „Obwohl bei $90 \%$ der Patienten eine gute Symptomkontrolle möglich wäre, leiden zu viele von ihnen an starken, oft unerträglichen Schmerzen", so Stefan Wirz, Sprecher des DGSS. Als Hauptgründe nannte er das Fehlen der Schmerztherapie als verpflichtendes Prüfungsfach in der medizinischen Approbationsordnung und die daraus resultierenden Vorbehalte und Ängste vor dem
Einsatz stark wirksamer Opioide. Für eine effektive Opioidtherapie ist bei schnell progredienten Tumorschmerzen ein Präparat wichtig, das flexibel an die zunehmende Schmerzintensität angepasst werden kann und das auch im Hochdosisbereich gut verträglich ist. Von Vorteil ist, wenn das Opioid in verschiedenen Applikationsformen verfügbar ist: So können umstellungsbedingte Nebenwirkungen vermieden werden, und das Umrechnen in Äquivalentdosen entfällt. Eine solche Schmerztherapie ist mit dem Hydromorphon Palladon ${ }^{\circledR}$ möglich, das in drei relevanten Darreichungsformen verfügbar ist: als Retardkapsel, als Rescuemedikation in verschiedenen Wirkstärken sowie als Injektionslösung in unterschiedlichen Ampullengrößen.

Mundipharma

\section{Magenkarzinom \\ Erste zielgerichtete Therapie}

\section{Für Patienten mit einme metastasierten HER2-positiven Adeno- karzinoms des Magens und des gastroösophagealen Übergangs steht nun eine neue Therapieoption für die Erstlinenbehanldung zur Verfügung: der monoklonale Antikörper Trastuzumab}

Der monoklonale Antikörper Trastuzumab (Herceptin $\left.{ }^{\circledR}\right)$ ist für die Erstlinienbehandlung des metastasierten HER2-positiven Adenokarzinoms des Magens und des gastroösophagealen Übergangs zugelassen worden. Damit steht für diesen Krebs erstmals eine zielgerichtete Therapie zur Verfügung, die das Überleben signifikant verlängert. Grundlage für die Zulas- sung sind die Ergebnisse der randomisierten offenen PhaseIII-Studie [Van Cutsem E et al. J Clin Oncol 2009; 27:18s, , suppl; abstr. LBA4509]. Darin wurden 3.807 Tumorbiopsien von Patienten, die zuvor keine Chemotherapie erhalten hatten, auf eine HER2-Überexpression überprüft. 584 Studienteilnehmer $(22,1 \%)$ konnten mittels Immunhistochemie (ICH3+ oder
IHC2+) und Fluoreszenz-in-situHybridisierung (FISH) positiv auf den HER2-Rezeptor getestet werden. Davon erhielt eine Gruppe Trastuzumab in Kombination mit Cisplatin plus Capecitabin oder 5-Fluorouracil, die zweite das gleiche Therapieregime ohne den Antikörper. Im Trastuzumab-Arm verlängerte sich das mediane Gesamtüberleben signifikant um 4,2 auf 16 Monate. Auch die sekundären Wirksamkeitsparameter wie z.B. das progressionsfreie Überleben wurden signifikant verbessert.

\section{RHA}

Quelle: Pressekonferenz am 17. Februar 2010 in Frankfurt/Main; Veranstalter: Roche Pharma 